\title{
Ginsenoside Re and Rd Enhance the Expression of Cholinergic Markers and Neuronal Differentiation in Neuro-2a Cells
}

\author{
Min Soo Kim, ${ }^{a, \#}$ Jung Min Yu, ${ }^{b, \#}$ Hee Jung Kim, ${ }^{b}$ Hae Bok Kim, ${ }^{b}$ Seung Tae Kim, ${ }^{b}$ Su Kil Jang, ${ }^{b}$ \\ Young Wook Choi, ${ }^{a}$ Do Ik Lee, ${ }^{a}$ and Seong Soo Joo*,b \\ ${ }^{a}$ College of Pharmacy, Chung-Ang University; 221 Heuksuk-dong, Dongjak-gu, Seoul 156-756, Republic of \\ Korea: and ${ }^{b}$ College of Life Science, Gangneung-Wonju National University; 120 Gangneung Daehangno, \\ Gangneung, Gangwon 210-702, Republic of Korea. \\ Received January 7, 2014; accepted February 27, 2014; advance publication released online March 5, 2014
}

In Alzheimer's disease (AD), extensive neuronal loss and a deficiency of the neurotransmitter acetylcholine (ACh) are the major characteristics during pathogenesis in the brain. In the present study, we aimed to investigate whether representative ginsenosides from ginseng can regulate choline acetyltransferase (ChAT) and vesicular acetylcholine transporter (VAChT), which are required for cholinergic neurotransmission. Our results revealed that $R e$ and $R d$ induced effectively the expression of ChAT/VAChT genes in Neuro-2a cells as well as ACh elevation. Microtubule-associated protein-2 (MAP-2), nerve growth factor receptor (p75), p21, and TrkA genes and proteins were also significantly expressed. Moreover, both activated extracelullar signalregulated protein kinase (ERK) and Akt were inhibited by K252a, a selective Trk receptor inhibitor. These findings strongly indicate that Re and Rd play an important role in neuronal differentiation and the nerve growth factor (NGF)-TrkA signaling pathway. High performance liquid chromatography analysis showed that $\mathrm{Re}$ and Rd administered orally were transported successfully into brain tissue and increased the level of ChAT and VAChT mRNA. The present study demonstrates that Re and Rd are selective candidates for upregulation of the expression of cholinergic markers, which may counter the symptoms and progress of AD.

Key words ginsenoside; choline acetyltransferase; vesicular acetylcholine transporter; acetylcholine; Alzheimer's disease

Neurodegenerative diseases, such as Alzheimer's disease (AD) or Parkinson's disease (PD), are incurable and debilitating conditions that result in progressive degeneration and/or death of nerve cells that primarily affect the neurons in the human brain. ${ }^{1)}$ Clinically, AD is characterized by low amounts of the enzyme choline acetyltransferase (ChAT) and enzyme abnormalities, which would produce reduced levels of the neurotransmitter acetylcholine (ACh). ${ }^{2)}$ ChAT activity and expression have been considered as markers for cholinergic neurons, which are involved in learning, memory, arousal, sleep, and movement. ${ }^{3)}$ Vesicular acetylcholine transporter (VAChT), which is responsible for the translocation of cytoplasmic acetylcholine synthesized by the catalytic activity of ChAT and involved in memory loss, is also specifically required for cholinergic neurotransmission. ${ }^{4,5)}$ Coexpression of these two proteins, which are colocalized within the genome and coregulated by various neurotrophic factors in neurons, is coordinately regulated and known to contribute significantly to the increase of intracellular $\mathrm{ACh}^{6}{ }^{6}$ Thus, expression of ChAT and of VAChT is required for the acquisition and the maintenance of the cholinergic phenotype after the induction of differentiation. ${ }^{7}$

Our previous study demonstrated that ChAT overexpressing human neural stem cells restored cognition in AF64A cholinotoxin rat model, resulting in an increasing ACh level in rat cerebrospinal fluid (CSF) ${ }^{8}{ }^{8}$ This implies that the increase of the ChAT level in cholinergic neurons plays a critical role in cognition enhancement and thus the cholinergic gene locus "VAChT/ChAT" can be a putative target for therapeutic approaches on AD. ${ }^{9-11)}$ Several studies reported that ginsenosides

The authors declare no conflict of interest

\# These authors contributed equally to this work. (i.e., $\mathrm{Rg} 1$ and $\mathrm{Rb} 1$ ) isolated from ginseng are responsible for ginseng's effects on the central nervous system (CNS) and the peripheral nervous system. ${ }^{12,13)}$ The objective of this study is to investigate whether five representative ginsenosides (Rb1, $\mathrm{Re}, \mathrm{Rd}, \mathrm{Rg} 1$, and Rg3) have beneficial effects on the cholinergic system and also to examine the possible mechanisms of the most active ginsenosides.

\section{MATERIALS AND METHODS}

Materials All chemicals were analytical grade. Ginsenosides (Rb1, Re, Rd, Rg1, and Rg3) derived from Panax ginseng at a purity of at least $95 \%$ were purchased from BTGin (Daejeon, Republic of Korea) (Fig. 1). A concentrated stock solution was prepared by dissolving each of the ginsenosides $(10 \mathrm{mg} / \mathrm{mL})$ in dimethylsulfoxide (DMSO, Sigma-Aldrich, St. Louis, MO, U.S.A.), and this was kept at $4^{\circ} \mathrm{C}$ until use. The stock solution was further diluted in culture media at $>1: 100$ ratio.

Cell Culture Neuro-2a (N2a) mouse neuroblastoma cells (Korean Cell Line Bank, Seoul, Republic of Korea) were grown in Dulbecco's modified Eagle's medium (DMEM) (Hyclone, Logan, UT, U.S.A.) supplemented with 10\% fetal bovine serum (FBS), $100 \mathrm{U} / \mathrm{mL}$ penicillin, and $100 \mu \mathrm{g} / \mathrm{mL}$ streptomycin (Invitrogen, Carlsbad, CA, U.S.A.). Cultures were maintained under $5 \% \mathrm{CO}_{2}$ at $37^{\circ} \mathrm{C}$ in tissue culture flasks. For all experiments, cells were grown to greater than $90 \%$ confluency and subjected to no more than 20 cell passages. The medium was changed every $2-3 d$. Subconfluent cells were harvested and seeded at a density of $5 \times 10^{5}$ cells or $5 \times 10^{6}$ cells in poly-L-lysine-coated $35 \mathrm{~mm}$ or $60 \mathrm{~mm}$ culture plates. After $48 \mathrm{~h}$ of plating, the medium was replaced with serum-free DMEM, washed once with phosphate buffered 


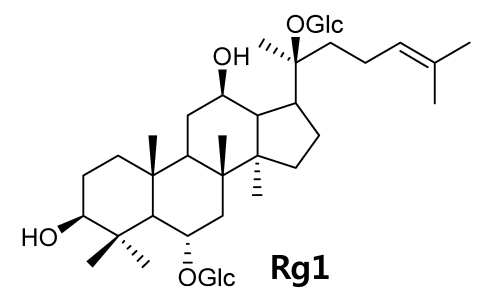

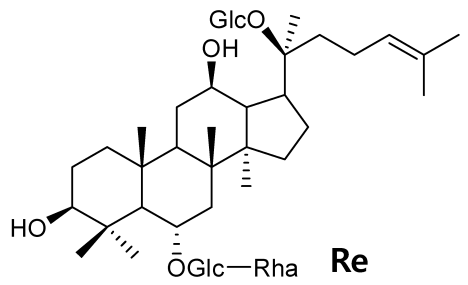

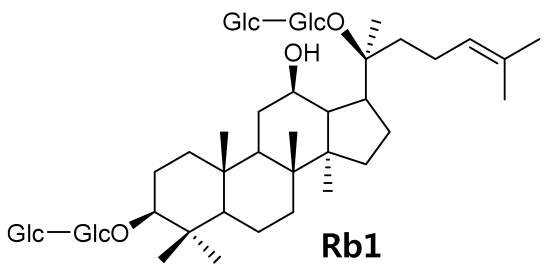

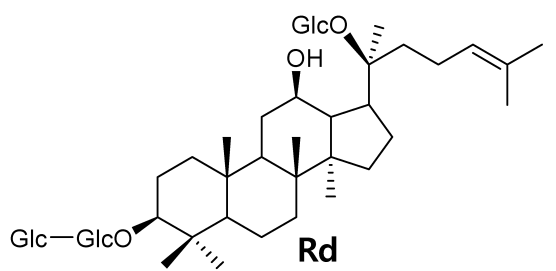<smiles>[R3][R3]</smiles>

Fig. 1. Molecular Structures of Ginsenosides, Rg1, Re, Rb1, Rd, and Rg3

Table 1. Primer Sequences Used for Real-Time or Conventional RT-PCR

\begin{tabular}{|c|c|c|c|c|}
\hline Gene & Primer & Amino acid sequences & Product size (bp) & Accession No. \\
\hline \multirow[t]{2}{*}{ ChAT } & 5'Primer & 5'-GTTCCCCAGAAACTCAAGGC & 280 & NM_009891 \\
\hline & 3'Primer & 5'-GAGTGGCCGATCTGATGTTG & & \\
\hline \multirow[t]{2}{*}{ VAChT } & 5'Primer & 5'-TTGATCGCATGAGCTACGAC & 246 & NM 021712 \\
\hline & 3'Primer & 5'-CCACTAGGCTTCCAAAGCTG & & \\
\hline \multirow[t]{2}{*}{ MAP2 } & 5'Primer & 5'-ACCACACCTGCAGTGGAGAA & 227 & M21041 \\
\hline & 3'Primer & 5'-AATCTGGACCTGGTTCCTGC & & \\
\hline \multirow[t]{2}{*}{ P21 } & 5'Primer & 5'-AGTGTGCCGTTGTCTCTTCG & 152 & AB017817 \\
\hline & $3^{\prime}$ Primer & 5'-TCAAAGTTCCACCGTTCTCG & & \\
\hline \multirow[t]{2}{*}{ TrkA } & 5'Primer & 5'-CCAGATGCCCAATGACTCTG & 215 & NM_001033124 \\
\hline & 3'Primer & 5'-CCCAGCACGTCACATTCTTC & & \\
\hline \multirow[t]{2}{*}{ NGF } & 5'Primer & 5'-TACTGCACCAATAGCTGCCC & 200 & M35075 \\
\hline & 3'Primer & 5'-TCCGGTGAGTCCTGTTGAAA & & \\
\hline \multirow[t]{2}{*}{ NGFR (P75) } & 5'Primer & 5'-AAGAGATCCCTGGTCGATGG & 226 & NM_012610 \\
\hline & 3'Primer & 5'-GCAGCCAAGATGGAGCAATA & & \\
\hline \multirow[t]{2}{*}{ CHT1 } & 5'Primer & 5'-TCTGCAGTACCTCTGCCCTG & 213 & AJ401467 \\
\hline & 3'Primer & 5'-GCTCCGAACACAAGCACAGT & & \\
\hline \multirow[t]{2}{*}{$\beta$-Actin } & 5'Primer & 5'-TACAGCTTCACCACCACAGC & 187 & NM_007393 \\
\hline & 3'Primer & 5'-AAGGAAGGCTGGAAAAGAGC & & \\
\hline
\end{tabular}

saline (PBS), and treated with Rb1, Re, Rd, Rg1, Rg3, or nerve growth factor (NGF, R\&D Systems, Minneapolis, MN, U.S.A.). Treatment with K252a (10 nM, Sigma-Aldrich) was used for inhibition of the specific inhibition of Trk receptor.

Quantitative Real-Time Polymerase Chain Reaction (PCR) Assay Total RNA extracts from N2a cells and perfused brain tissues (50 mg of cerebral cortices) were prepared using the Trizol method (Invitrogen). cDNA was synthesized from RNA by reverse transcription of $1 \mu \mathrm{g}$ of total RNA using the Improm-II reverse transcription system (Promega, Madison, WI, U.S.A.) and oligo dT primers in a total volume of $20 \mu \mathrm{L}$. PCR amplification was performed using the primers described in Table 1 (Bioneer, Deajeon, Korea). Quantitative real-time PCR reactions were run on a Rotor-Gene 6000 (Corbett Research, Sydney, Australia) using SensiMix ${ }^{\mathrm{TM}}$ SYBR Hi-ROX Kit (Bioline, London, U.K.) in $20 \mu \mathrm{L}$ reaction 
mixtures. Each real-time-PCR master mix contained $10 \mu \mathrm{L}$ $2 \times$ enzyme mastermix, $7.0 \mu \mathrm{L}$ RNase free water, $1 \mu \mathrm{L}$ of each primer (10 pm each), and $1 \mu \mathrm{L}$ diluted template. The PCR was performed with an initial pre-incubation step for $15 \mathrm{~min}$ at $95^{\circ} \mathrm{C}$, followed by 45 cycles of $95^{\circ} \mathrm{C}$ for $15 \mathrm{~s}$, annealing at $52^{\circ} \mathrm{C}$ for $15 \mathrm{~s}$, and extension at $72^{\circ} \mathrm{C}$ for $10 \mathrm{~s}$. Melting curve analysis was used to confirm formation of the expected PCR product, and products from all assays were additionally tested with $1.2 \%$ agarose gel electrophoresis to confirm the correct lengths. An inter-run calibrator was used, and a standard curve was created for each gene to obtain PCR efficiencies. Relative sample expression levels were calculated using RotorGene 6000 Series Software 1.7, and were expressed relative to $\beta$-actin and corrected for between-run variability. Data for the experimental samples were expressed as a fold unit the internal control gene.

Western Blot Analysis N2a cells were lysed in 1\% radioimmunoprecipitation assay (RIPA) buffer containing protease and phosphatase inhibitors (Roche, Mannheim, Germany) and whole cell lysates were separated by $10 \%$ sodium dodecyl sulfate polyacrylamide gel electrophoresis (SDS-PAGE). After electrophoresis, proteins were transferred onto polyvinylidene fluoride (PVDF) membranes and the membranes were blocked with $5 \%$ skim milk in Tris-buffered saline solution containing $0.1 \%$ Tween-20. The membrane was then immunoblotted with primary antibodies, anti-phospho-Akt, antiphospho-extracelullar signal-regulated protein kinase (ERK), anti-ChAT and anti- $\beta$-actin (Santa Cruz Biotechnology, Santa Cruz, CA, U.S.A.), and this was followed by incubation with horseradish peroxidase-conjugated anti-rabbit or anti-mouse secondary antibodies (Stressgen, San Diego, CA, U.S.A.). Blots were developed using an enhanced chemiluminescence (ECL) solution (Thermo, Rockford, IL, U.S.A.).

Immunocytochemistry (ICC) and Microscopic Observation Cultured N2a cells were fixed in 4\% paraformaldehyde (in PBS) for $15 \mathrm{~min}$, washed twice with PBS supplemented with $100 \mathrm{~mm}$ glycine for $5 \mathrm{~min}$, and incubated with permeabilization buffer consisting of $0.1 \%$ Triton X-100 (Sigma-Aldrich) in $\mathrm{PBS}$ for $30 \mathrm{~min}$ at room temperature. Blocking was performed with $1 \%$ bovine serum albumin (BSA, Sigma-Aldrich) for $30 \mathrm{~min}$ at room temperature. Then Microtubule-Associated Protein-2 (MAP-2) or VAChT mouse monoclonal antibody 1:200 (Santa Cruz Biotechnology) was added to $1 \%$ BSA in PBS with Tween 20 (PBST) and incubated for $2 \mathrm{~h}$ at room temperature. Cells were then washed three times with PBS before fluorescein isothiocyanate (FITC)-conjugated antimouse immunoglobulin G (IgG) 1:200 (Cell Signaling Technology, Danvers, MA, U.S.A.) was added to $1 \%$ BSA for $1 \mathrm{~h}$ at room temperature. The cells were rinsed and counterstained with 4',6-diamidino-2-phenylindole (DAPI, Sigma-Aldrich) for $10 \mathrm{~min}$, followed by two PBS washes. Cultures were visualized with an inverted fluorescent microscope system (Eclipse Ti-S; Nikon, Tokyo, Japan) at a magnification of $\times 600$ and optical inverted microscopy (OLYMPUS CKX41, Tokyo, Japan) at a magnification of $\times 200$.

Colorimetric Acetylcholine Measurement Quantitation of acetylcholine in cultured media was measured using a commercial acetylcholine assay kit (Cell Biolabs, San Diego, CA, U.S.A.), based on the enzyme driven reaction that will detect acetylcholine via acetylcholinesterase enzyme and choline oxidase. For the measurement, a reaction reagent was pre- pared by combining $12.5 \mu \mathrm{L}$ of choline oxidase, $5 \mu \mathrm{L}$ of horse radish peroxidase, $50 \mu \mathrm{L}$ of colorimetric probe, and $10 \mu \mathrm{L}$ of acetylcholinesterase with $1 \mathrm{X}$ assay buffer to $2.5 \mathrm{~mL}$ total solution. After incubation with the mixture of $50 \mu \mathrm{L}$ of prepared acetylcholine reaction reagent and the same amount of culture media on an orbital rotator for $1 \mathrm{~h}$ at room temperature, the acetylcholine levels were quantified spectrophotometrically by measuring the absorbance at $540 \mathrm{~nm}$ (Molecular Devices, Sunnyvale, CA, U.S.A.).

High Performance Liquid Chromatography (HPLC) Analysis For the HPLC analysis of ginsenosides transported into the brain, a mixture of Rb1, Re, Rd, Rg1, and Rg3 (10 mg/ $\mathrm{kg}$ each) was administered to ten-week-old male BALB/c mice (Central Lab. Animal, Seoul, Republic of Korea). Brain tissues were isolated six hours after the oral administration of the ginsenosides after the complete perfusion with $0.9 \%$ saline solution, mechanically disrupted using a TissueLyser (Qiagen, Crawley, U.K.), and centrifuged for $10 \mathrm{~min}$ at $13500 \mathrm{rpm}$. The supernatant $(1 \mathrm{~mL})$ was then mixed with $n$-butanol $(0.4 \mathrm{~mL})$ and the butanol layer was completely evaporated and dried for HPLC analysis. The chromatography was performed by the Agilent 1100 series HPLC system (Agilent Technologies, Palo Alto, CA, U.S.A.) equipped with a vacuum degasser, quaternary pump, and ultraviolet (UV) detector. Chromatographic separation was performed on a Zorbax SB-Aq C18 column $(4.6150 \mathrm{~mm}, 5 \mu \mathrm{m})$ and a mixture of solvent A (water) and solvent B (acetonitrile) flowed at a rate of $1 \mathrm{~mL} / \mathrm{min}$. The gradient elution program was initiated with $79 \%$ solvent $\mathrm{A}$ and $21 \%$ solvent $\mathrm{B}, 0-10 \mathrm{~min}(21-22 \% \mathrm{~B}), 10-11 \mathrm{~min}(22-23 \%$ B), $11-29 \min (23-24 \% \mathrm{~B}), 29-34 \mathrm{~min}(24-30 \% \mathrm{~B}), 34-44 \mathrm{~min}$ $(30-32 \%$ B), $44-49 \mathrm{~min}(32-50 \%$ B), 49-64 min $(50-65 \%$ B), 64-78 $\min (65-100 \% \mathrm{~B})$, and equilibrated with the initial gradient program $(21 \% \mathrm{~B})$ for $10 \mathrm{~min}$ prior to the next injection. The column temperature was set at $35^{\circ} \mathrm{C}$, the volume of the sample injection was $10 \mu \mathrm{L}$, and detection wavelength was set at $203 \mathrm{~nm}$. The animal experiments were approved by the Gangneung-Wonju National University Animal Care and Use Committee (Approval Number: GWNU-2013-23), and all procedures were conducted in accordance with the Guide for Care and Use of Laboratory Animals published by the National Institute of Health.

Statistical Analysis Statistical comparisons between the groups were performed using one-way ANOVA with a Dunnet's post-hoc test using SPSS software (v. 13) (Chicago, IL, U.S.A.). Statistical significance was set a priori at $p<0.05$.

\section{RESULTS}

Effect of Ginsenosides on the Cholinergic Markers in N2a Cells In our earlier study, we reported that ChAT overexpressing human neural stem cells restored cognition with an increase of ACh levels in rat model. ${ }^{8)}$ In line with this, we evaluated whether the ginsenosides increase ChAT and VAChT expression that are required for cholinergic neurotransmission, such as ACh. In our system, the expression of ChAT and VAChT mRNA in N2a cells was significantly increased in case of treatment with $\mathrm{Re}$ and $\mathrm{Rd}$, while other ginsenosides (Rb1, Rg1, and Rg3) showed no significant increase (Figs. 2A, B), even though Rb1 facilitates the release of acetylcholine (ACh) from hippocampal slices in a previous study. ${ }^{14)}$ We also found significant increases of mRNA levels 

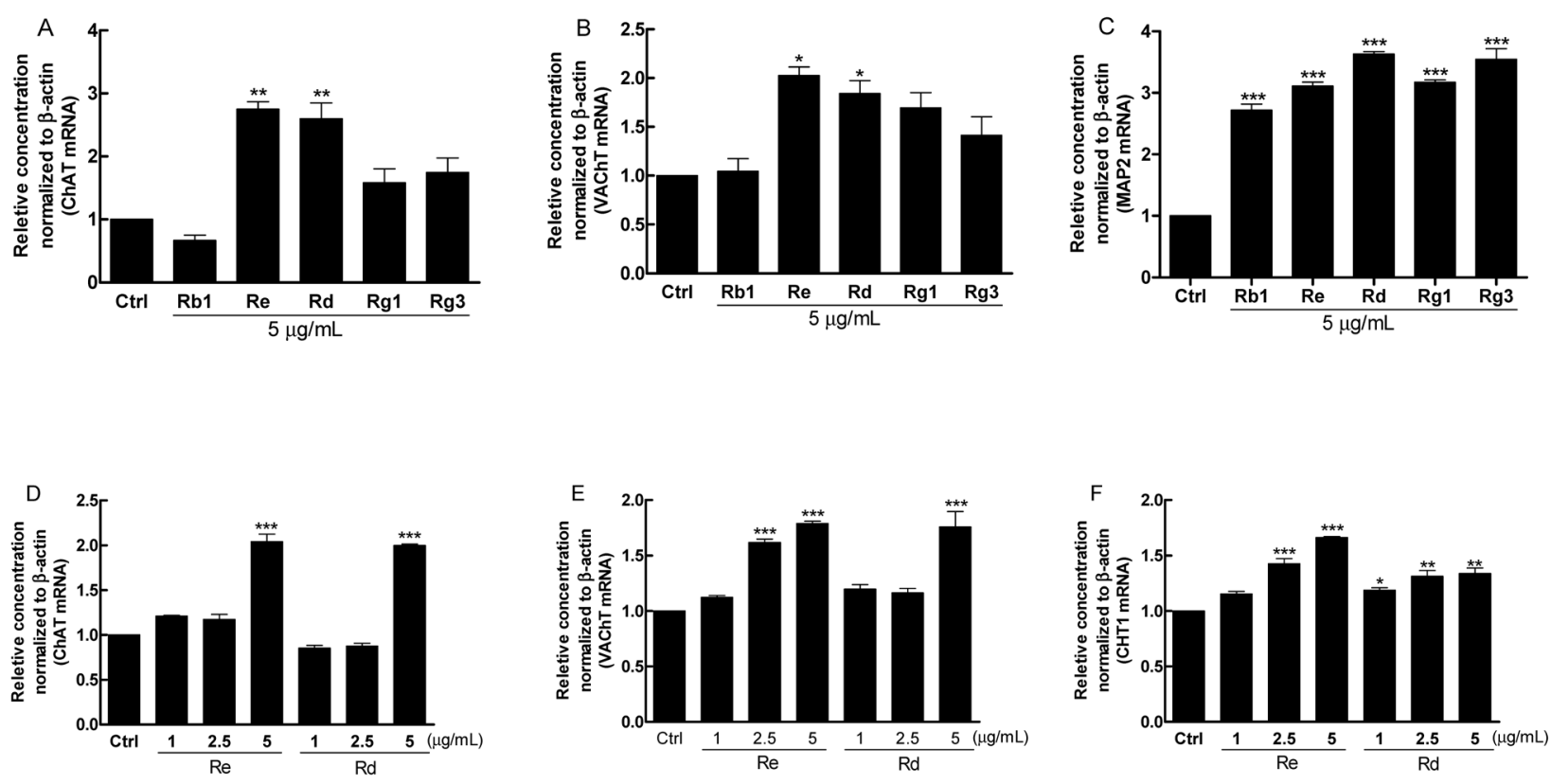

Fig. 2. Effects on ChAT/VAChT/MAP-2/CHT1 Gene Expression

N2a cells were seeded on $35 \mathrm{~mm}$ culture plates and treated with Rb1, Re, Rd, Rg1, or Rg3 for 48h. ChAT/VAChT/MAP-2/CHT1 mRNAs were quantified by fold unit using real-time PCR as described in Materials and Methods. Each ginsenoside treatment was $5 \mu \mathrm{g} / \mathrm{mL}(\mathrm{A}-\mathrm{C}$ ), while ginsenosides Re and Rd were also dose-dependently observed ( 1 to $5 \mu \mathrm{g} / \mathrm{mL}$ ) (D-F). Results are expressed as means \pm standard deviations (S.D.) from three separate experiments. ${ }^{*} p<0.05$, ${ }^{* *} p<0.01$, ${ }^{* * *} p<0.001 v$. Ctrl.
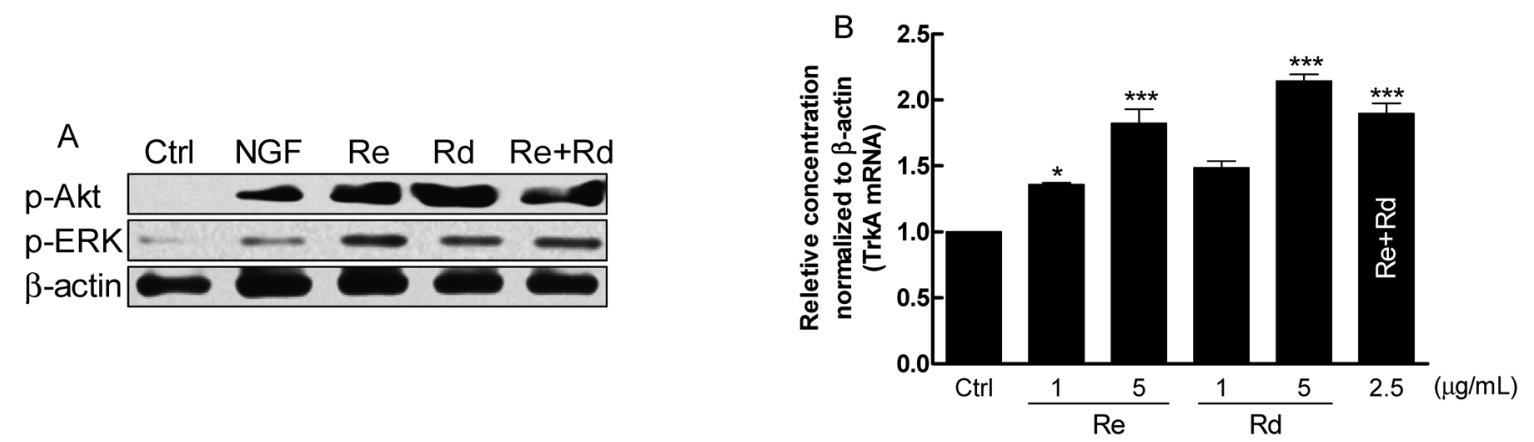

Fig. 3. Western Blot Analysis of Erk and Akt Phosphorylation

Immunoblotting analysis of phospho-ERK and -Akt protein levels was investigated in the presence of NGF or Re/Rd (A). Cells were seeded on $60 \mathrm{~mm}$ culture dishes and treated with NGF $(100 \mathrm{ng} / \mathrm{mL})$, Re $(5 \mu \mathrm{g} / \mathrm{mL})$, or Rd $(5 \mu \mathrm{g} / \mathrm{mL})$ and incubated for $1 \mathrm{~h}$ in phenol red- /FBS-free DMEM media. For TrkA mRNA expression, Re, Rd, and $\mathrm{Re} / \mathrm{Rd}$ were treated for $24 \mathrm{~h}$ and analyzed using real-time PCR (B). Results are expressed as means \pm S.D. from three separate experiments. ${ }^{*} p<0.05, * * * p<0.001 v s$. Ctrl.

of MAP-2 in five ginsenoside samples (Fig. 2C), while ChAT and VAChT mRNA levels were increased by $\mathrm{Re}$ or $\mathrm{Rd}$ at doses up to 2.5 to $5 \mu \mathrm{g} / \mathrm{mL}$ (Figs. 2D, E). Moreover, the highaffinity choline transporter CHT1 mRNA, which is responsible for choline uptake, ${ }^{15)}$ was increased when treated with $\mathrm{Re}$ and Rd (Fig. 2F).

Activation of ERK and Akt Signaling by Ginsenosides Re and Rd Two major cholinergic markers, ChAT and VAChT, are known to be regulated via the NGF-TrkA signaling pathway, which activates ERK and Akt downstream pathways for cholinergic gene expression. ${ }^{16,17)}$ Figure 3A shows that Re and Rd could activate ERK and Akt with phosphorylation as much as NGF, suggesting that Re and Rd are closely related with the NGF-TrkA signaling pathway (Fig. 3B). This activation was coincidently observed with co-treatment with Re and Rd.

Expression of MAP-2/p75/ChAT/VAChT and the Neuronal Differentiation of N2a Cells ChAT is the important enzyme involved in the synthesis of the neurotransmitter acetylcholine, and thus it is a marker for differentiated cholinergic neurons. Based on this, we assumed that general neuron marker (e.g. MAP-2) and the earliest cholinergic surface markers (e.g. p75) should be expressed together with cholinergic cell markers such as the enzyme ChAT and VAChT. ${ }^{18,19)}$ Our result revealed that $\mathrm{Re}$ and $\mathrm{Rd}$ stimulated effectively the production of MAP-2 protein and switched to p75-positive N2a cells to the same or more extent as NGF (Figs. 4A, B). Figure $4 \mathrm{C}$ shows the morphological changes of $\mathrm{N} 2 \mathrm{a}$ cells induced by $\mathrm{NGF}, \mathrm{Re}$, and Rd. In case N2a cells were cultured without any treatment, the shape was mostly rounded; whereas cells treated with NGF, Re, and Rd induced the production of distinctive multiple projections (club shapes). Notably, MAP-2 mRNA was significantly expressed by $\mathrm{Re}$ and $\mathrm{Rd}$ as shown in Fig. 4D. As expected, ChAT and VAChT were distinctively expressed in the presence of $\mathrm{Re}$ and Rd (Figs. 5A, B), suggesting a functional contribution of $\mathrm{Re}$ and $\mathrm{Rd}$ in the neuronal differentiation and cholinergic gene expression of N2a cells.

Inhibition of NGF-Induced Trk Signaling Pathway in 

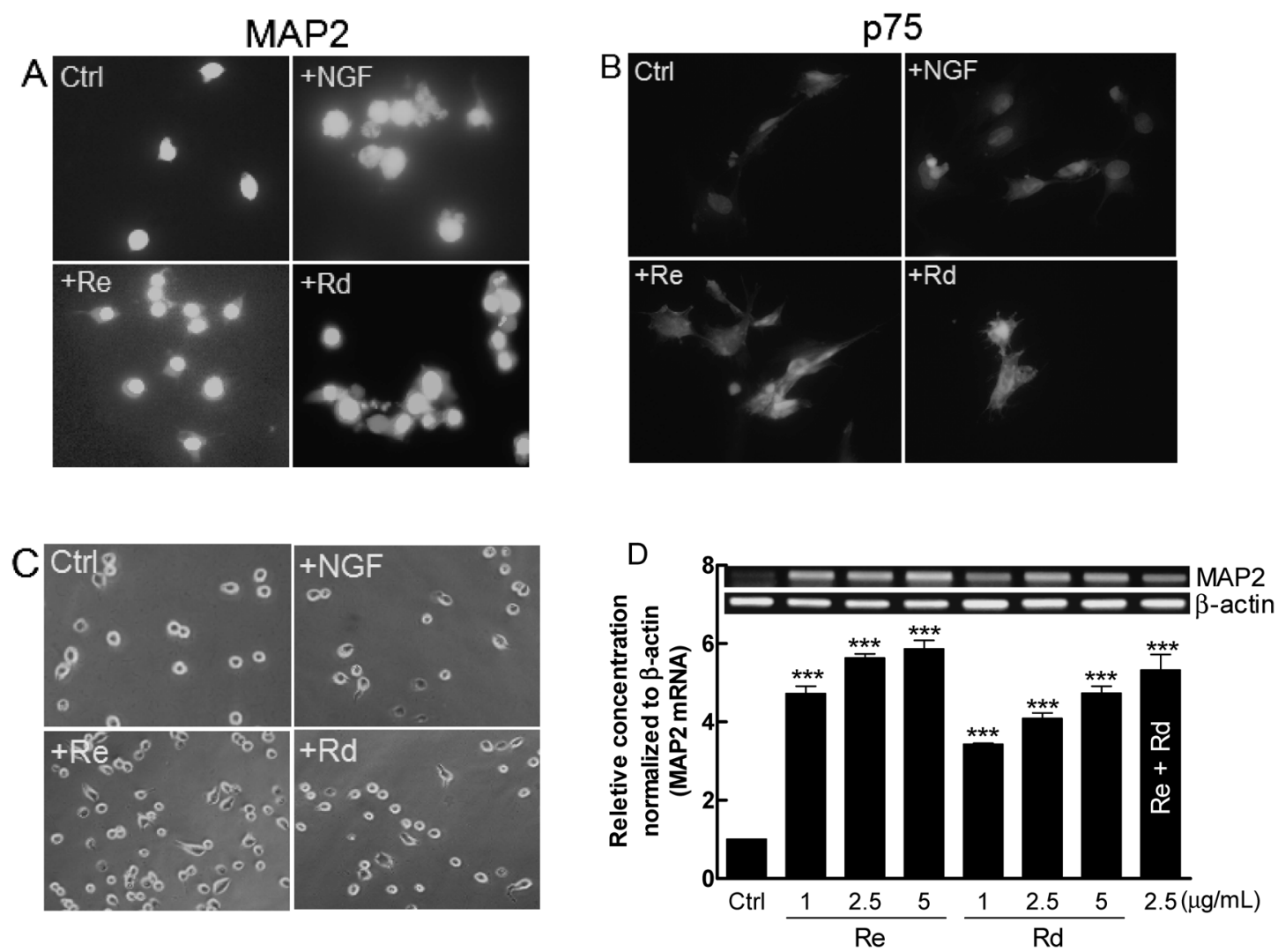

Fig. 4. MAP-2/p75 Expression and Morphological Changes of N2a Cells

Expression of neuronal marker (MAP-2) and the earliest cholinergic surface marker (p75) induced by NGF, Re, or Rd was analyzed after 48h. N2a cells were cultured in chamber slides in phenol red-/FBS-free DMEM media for $48 \mathrm{~h}$ and immunostained with FITC-conjugated anti-MAP-2 or p-75 (green), followed by DAPI for DNA staining (blue). MAP-2 and p-75 expression was investigated after merging two pictures from MAP-2 or p75 and DAPI staining at the same location (A, B). Neurite outgrowth (club shapes) was determined under the optical inverted microscopy system (C). MAP-2 mRNA expression was quantified under the same culture conditions (D). Results are expressed as means \pm S.D. from three separate experiments. $* * * p<0.001$ vs. Ctrl. (Color images were converted into gray scale.)
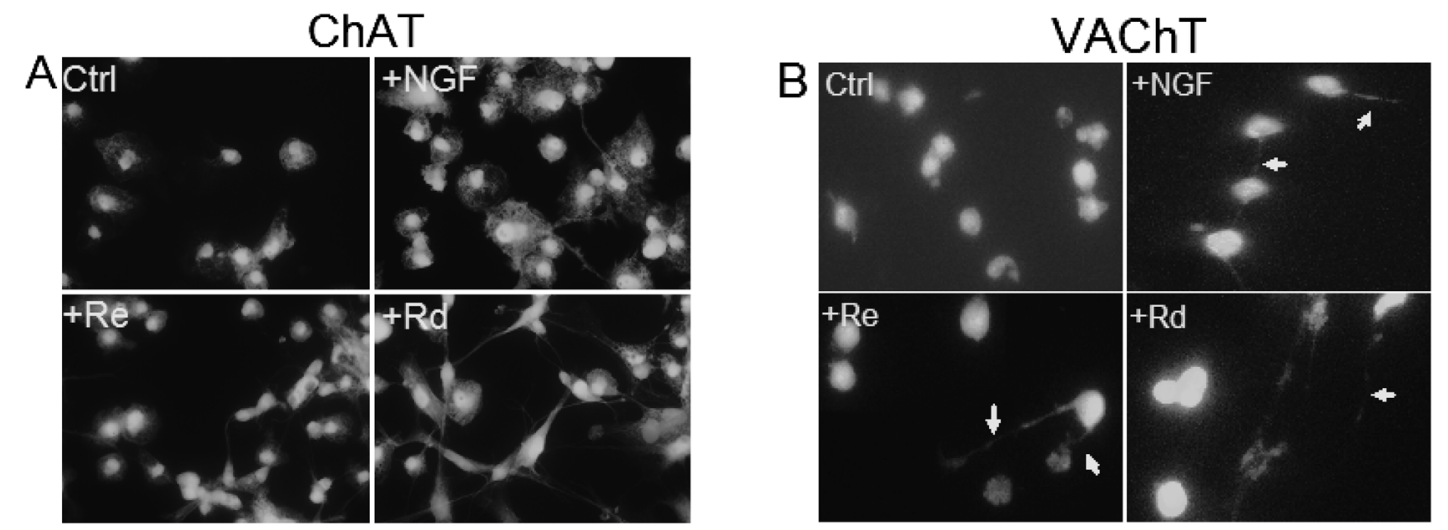

Fig. 5. Immunostaining of ChAT and VAChT in N2a Cells

ICC shows that cholinergic markers, ChAT, were remarkably expressed (A) and VAChT proteins were distinctively transported to termini of the projections (arrows) (B).

N2a Cells K252a, which has potent kinase C and Trk inhibitory activities, is known to block NGF-activated increases in ChAT mRNA and activity. ${ }^{20)}$ As NGF-Trk signaling upregulates ChAT and VAChT, it is very important to know whether $\mathrm{Re}$ and $\mathrm{Rd}$ have direct or at least indirect association with Trk activity. ${ }^{21)}$ Our work showed that ChAT expression in N2a cells was inhibited in case of co-treatment with K252a+Re or $+\mathrm{Rd}$ compared with $\mathrm{Re}$ and $\mathrm{Rd}$ single treatments, suggesting that $\mathrm{Re}$ and $\mathrm{Rd}$ are related to the Trk signaling pathway (Fig. 6A). In addition, NGF and NGFR (p75) mRNA levels were significantly increased when treated with Re and Rd (Fig. 6B).
Figures $6 \mathrm{C}$ and D revealed that ChAT and p21 mRNA expression were significantly reduced in the $\mathrm{Re}$ and $\mathrm{Rd}$ groups that were pre-treated with $\mathrm{K} 252 \mathrm{a}$, whereas ACh production was increased with $\mathrm{Re}$ or $\mathrm{Rd}$ alone (Fig. 6E). Together, these results indicate that $\mathrm{Re}$ and $\mathrm{Rd}$ have a potent activity in increasing the neurotransmitter ACh via NGF mimic signal transduction.

Determination of Ginsenosides in Brain Tissue by HPLC Analysis The blood-brain barrier (BBB) is a major site for controlling the entry of substances into the brain. Thus, it is very important to investigate whether or not the studied five ginsenosides have the capability to penetrate the blood-brain 

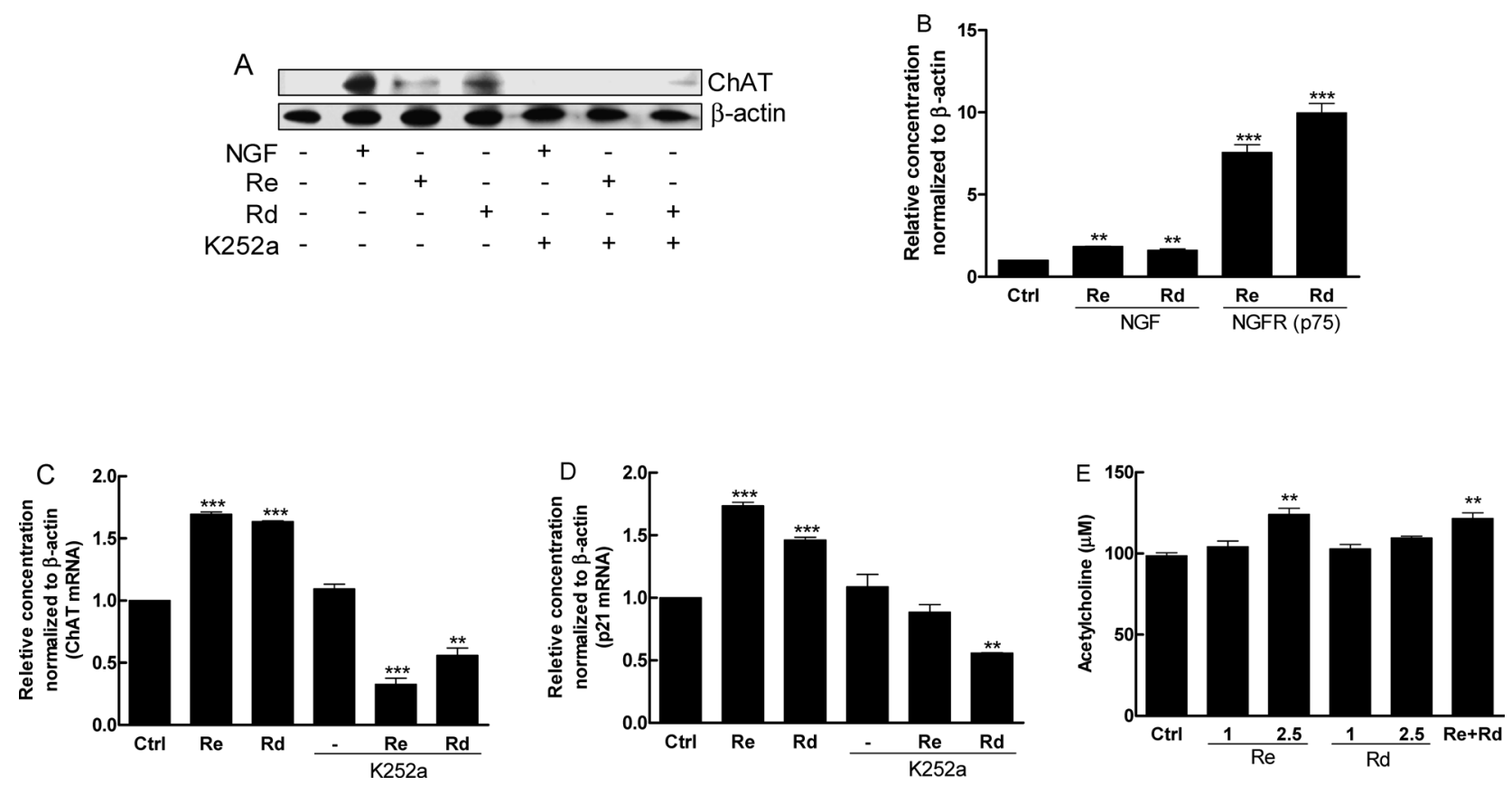

Fig. 6. Inhibition of Trk Pathway via Trk Inhibitor, K252a

N2a cells were treated with or without K252a ( $50 \mathrm{~nm})$ in the presence of Re $(5 \mu \mathrm{g} / \mathrm{mL})$ or Rd $(5 \mu \mathrm{g} / \mathrm{mL})$ for $48 \mathrm{~h}$. Total RNA and protein lysates were isolated as described in Materials and Methods for analysis of protein (A) and mRNA (B-D) expression under the same conditions. Acetylcholine production was determined from the culture media (E). $* * p<0.01, * * * p<0.001$ vs. Ctrl.
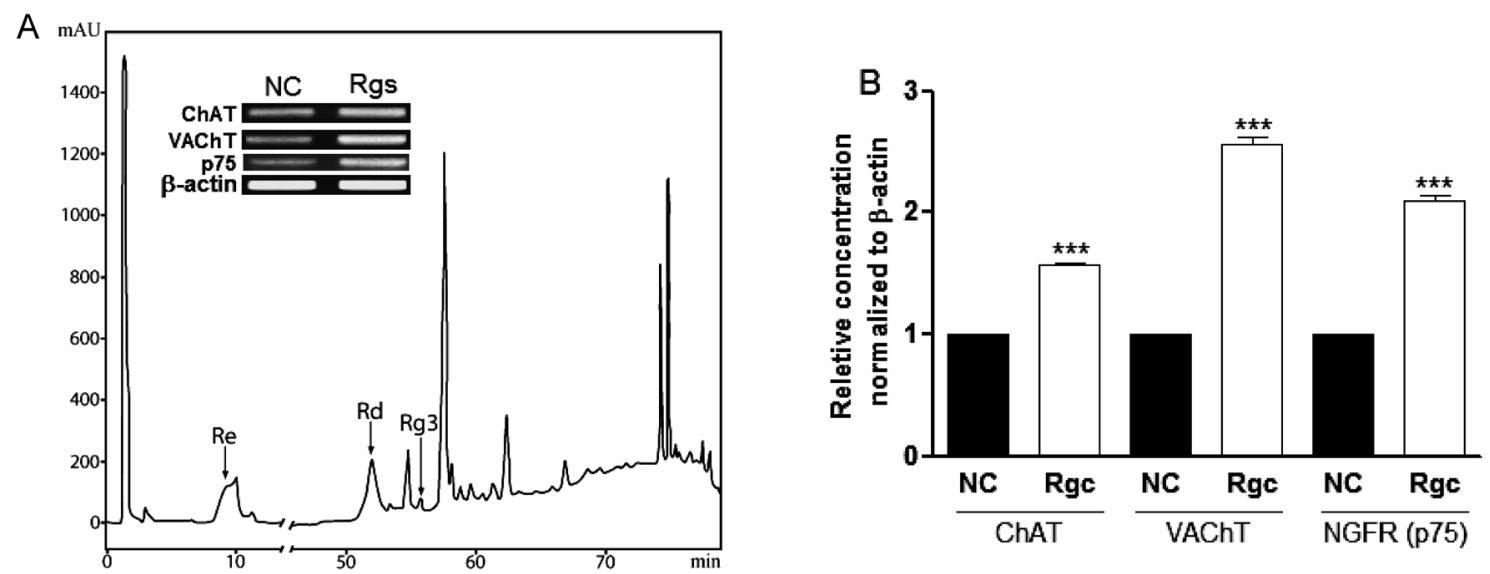

Fig. 7. HPLC Analysis of Ginsenosides and ChAT/VAChT/NGFR (p75) Gene Expression in Brain Tissue

HPLC analysis of perfused brain confirmed transport of ginsenosides Re, Rd, and Rg3 six hours after the oral administration of five ginsenosides. ChAT, VAChT and NGFR (p75) mRNA expressions were analyzed in the same brain tissue (upper left) (A). ChAT/VAChT/NGFR (p75) mRNA levels were quantified using real-time PCR (B). NC: normal control; 5Gs: Rb1, Re, Rd, Rg1, and Rg3. Results are expressed as means \pm S.D. from three separate experiments. *** $p<0.001 v s$. Ctrl (each).

barrier. HPLC analysis clearly confirmed that three ginsenosides ( $\mathrm{Re}, \mathrm{Rd}$, and $\mathrm{Rg} 3$ ) out of the five penetrated into the brain (Fig. 7). There was a remarkable increase in ChAT and VAChT mRNA from the same brain tissue six hours after oral administration (upper-left). Interestingly, another cholinergicspecific gene, p75, was significantly increased in the brain cortex.

\section{DISCUSSION}

The major characteristics of $\mathrm{AD}$ are cholinergic abnormalities, senile plaques, neurofibrillary tangles, and extensive neuronal loss, and it is closely linked to a deficiency of the neurotransmitter ACh during pathogenesis in the brain. Thus, the cholinergic deficiency hypothesis has long been the basis for therapeutic strategies and drug development for $A D{ }^{22)}$ In line with this, increases in ChAT activity and ACh synthesis, which are strongly correlated with the degree of cognitive impairment in patients with $\mathrm{AD}$, are major areas of interest for new AD therapies. Our previous study revealed that human neural stem cells transfected with ChAT-containing vector significantly enhanced the cognitive functions in rat models. In this paper, we present data suggesting that $\mathrm{Re}$ and $\mathrm{Rd}$ out of five representative ginsenosides isolated from ginseng deserve to be developed as new promising candidates for enhancement of cognition via increasing ChAT/VAChT expression, and thereby elevating $\mathrm{ACh}$ in the N2a neuroblastoma cell model. As cholinergic neurons are known to coexpress ChAT and VAChT proteins, ${ }^{23)}$ MAP-2, a neuronal differentiation marker, was also included in this study. ${ }^{24}$ 
Using N2a cells, we demonstrated that Re and Rd were highly promising candidates for the enhancement of the cholinergic system, which is severely impaired in patients with AD. Data showed that $\mathrm{Re}$ and $\mathrm{Rd}$ significantly increased the expression of two major cholinergic markers, ChAT and VAhT, in N2a cells compared with the other tested ginsenosides, Rb1, Rg1, and Rg3, indicating that Re and Rd might be directly or indirectly associated with the cholinergic gene locus. Since VAChT, a reliable and specific marker to identify cholinergic synapses, is colocalized with the corresponding protein (ChAT), VAChT is a more specific marker to reveal cholinergic networks than acetylcholinesterase. ${ }^{25)}$ Our results suggest that Re and Rd are highly associated with the NGFinduced signaling pathway in terms of activation of ERK and Akt, which are known to respond to NGF via the Trk receptor and to induce upregulation of ChAT and VAChT expression including neuronal differentiation. ${ }^{26,27)}$ In these investigations, we used N2a cells derived from spontaneous neuroblastoma of mouse, which are capable of differentiation into neuronal-like cells. $^{28)}$

ChAT and VAChT proteins are only synthesized in cholinergic cells in the nervous system and their expression is tightly linked. ${ }^{29)}$ In this regard, we demonstrated that MAP-2, a neuronal differentiation marker, and the nerve growth factor receptor (p75), a surface marker for mature basal forebrain cholinergic neurons, were specifically induced by $\mathrm{Re}$ and $\mathrm{Rd}$ treatment. Also from both fluorescence and optical observations for MAP-2 we detected distinctive neurite outgrowth from Re/Rd-treated N2a cells that was similar to that shown by treatment with NGF, indicating successful differentiation into neuronal-like cells. Moreover, TrkA, NGF and NGFR (p75) mRNA was significantly increased in the presence of $\mathrm{Re}$ or $\mathrm{Rd}$, indicating the involvement of neuronal differentiation via NGF mimic signal transduction. ${ }^{30)}$ Another interesting result was the increase of $\mathrm{p} 21$, a cyclin-dependent kinase inhibitor, because p21 is known to arrest cell cycle progression and to promote neuronal differentiation. ${ }^{31)}$ The significant increase of p21 by $\mathrm{Re}$ and $\mathrm{Rd}$ was lessened in case of pretreatment with K252a, indicating Trk NGF receptor tyrosine kinase lacks NGF-inducible p21 activation in association with neuronal differentiation. ${ }^{32)}$ Elevation of $\mathrm{ACh}$ production from $\mathrm{N} 2 \mathrm{a}$ cell suggests that $\mathrm{Re}$ and Rd may contribute to the synthesis of ACh via increasing ChAT/VAChT expression after neuronal-like differentiation. ${ }^{33)}$

As all CNS pharmaceuticals should be capable of crossing the $\mathrm{BBB}$, it is very important to determine whether or not the developed anti-AD candidates pass through the BBB, naturally or artificially with appropriate lipid meditation. Lipid-soluble small molecules with a molecular mass under $400-600 \mathrm{Da}$ can readily be transported through the BBB. ${ }^{34)}$ Several reports have indicated that ginsenosides are only poorly absorbed from digestive tracts because of their molecular sizes $(>600)$ and hydrophilicity. ${ }^{35,36)}$ Regardless, HPLC analysis demonstrated that $\mathrm{Re}, \mathrm{Rd}$, and $\mathrm{Rg} 3$, after oral administration, were transported into brain tissue and increased the level of ChAT and VAChT mRNA, which coincided with previous reports. ${ }^{37,38)}$ There is no concrete evidence or known mechanisms for the activation of NGF-induced Trk signal transduction via $\mathrm{Re}$ and $\mathrm{Rd}$; however, one possibility is that ginsenosides have an estrogen-like effect, ${ }^{39)}$ whereby estrogen may stimulate the synthesis of proteins required for neuronal differentiation, survival, and maintenance of function via up-regulation of TrkA mRNA in cholinergic neurons as well as upregulation of ChAT and VAChT genes, which share the same cholinergic locus. ${ }^{7,40)}$

Taken together, we concluded that ginsenosides $\mathrm{Re}$ and Rd enhance effectively ChAT and VAChT expression in N2a cells, followed by an increase of ACh production that may counter the symptoms and progress of AD. Although in-depth mechanism studies are required for more concrete information on the pharmacological features of $\mathrm{Re}$ and $\mathrm{Rd}$, our findings strongly support the conclusion that the selective ginsenosides $\mathrm{Re}$ and $\mathrm{Rd}$ contribute to differentiation into neuronal-like cells via cell cycle regulation.

Acknowledgment This research was supported by Basic Science Research Program through the National Research Foundation of Korea (NRF) funded by the Ministry of Education, Science and Technology (NRF-2012R1A1A2007240).

\section{REFERENCES}

1) Esposito E, Cuzzocrea S. New therapeutic strategy for Parkinson's and Alzheimer's disease. Curr. Med. Chem., 17, 2764-2774 (2010).

2) Giacobini E. Cholinergic function and Alzheimer's disease. Int. J. Geriatr. Psychiatry, 18 (Suppl. 1), S1-S5 (2003).

3) Oda Y. Choline acetyltransferase: The structure, distribution and pathologic changes in the central nervous system. Pathol. Int., 49, 921-937 (1999).

4) Roghani A, Carroll PT. Analysis of uptake and release of newly synthesized acetylcholine in PC12 cells overexpressing the rat vesicular acetylcholine transporter (VAChT). Brain Res. Mol. Brain Res., 100, 21-30 (2002).

5) Chen KH, Reese EA, Kim H-W, Rapoport SI, Rao JS. Disturbed neurotransmitter transporter expression in Alzheimer disease brain. J. Alzheimers Dis., 26, 755-766 (2011).

6) Berse B, Blusztajn JK. Coordinated up-regulation of choline acetyltransferase and vesicular acetylcholine transporter gene expression by the retinoic acid receptor alpha, cAMP, and leukemia inhibitory factor/ciliary neurotrophic factor signaling pathways in a murine septal cell line. J. Biol. Chem., 270, 22101-22104 (1995).

7) Yamamuro Y, Aizawa S. Asymmetric regulation by estrogen at the cholinergic gene locus in differentiated NG108-15 neuronal cells. Life Sci., 86, 839-843 (2010).

8) Park D, Lee HJ, Joo SS, Bae DK, Yang G, Yang YH, Lim I, Matsuo A, Tooyama I, Kim YB, Kim SU. Human neural stem cells overexpressing choline acetyltransferase restore cognition in rat model of cognitive dysfunction. Exp. Neurol., 234, 521-526 (2012).

9) Yamada M, Chiba T, Sasabe J, Terashita K, Aiso S, Matsuoka M. Nasal Colivelin treatment ameliorates memory impairment related to Alzheimer's disease. Neuropsychopharmacology, 33, 2020-2032 (2008).

10) Counts SE, He B, Che S, Ginsberg SD, Mufson EJ. Galanin hyperinnervation upregulates choline acetyltransferase expression in cholinergic basal forebrain neurons in Alzheimer's disease. Neurodegener. Dis., 5, 228-231 (2008).

11) Fukasawa H, Nakagomi M, Yamagata N, Katsuki H, Kawahara K, Kitaoka K, Miki T, Shudo K. Tamibarotene: A candidate retinoid drug for Alzheimer's disease. Biol. Pharm. Bull., 35, 1206-1212 (2012).

12) Nah SY, Kim DH, Rhim H. Ginsenosides: are any of them candidates for drugs acting on the central nervous system? CNS Drug Rev., 13, 381-404 (2007).

13) Chang Y, Huang WJ, Tien LT, Wang SJ. Ginsenosides Rg1 and Rb1 enhance glutamate release through activation of protein kinase A in 
rat cerebrocortical nerve terminals (synaptosomes). Eur. J. Pharmacol., 578, 28-36 (2008).

14) Benishin CG, Lee R, Wang LC, Liu HJ. Effects of ginsenoside Rb1 on central cholinergic metabolism. Pharmacology, 42, 223-229 (1991).

15) Okuda T, Konishi A, Misawa H, Haga T. Substrate-induced internalization of the high-affinity choline transporter. J. Neurosci., 31, 14989-14997 (2011).

16) Williams B, Granholm AC, Sambamurti K. Age-dependent loss of NGF signaling in the rat basal forebrain is due to disrupted MAPK activation. Neurosci. Lett., 413, 110-114 (2007).

17) Madziar B, Shah S, Brock M, Burke R, Lopez-Coviella I, Nickel AC, Cakal EB, Blusztajn JK, Berse B. Nerve growth factor regulates the expression of the cholinergic locus and the high-affinity choline transporter via the Akt/PKB signaling pathway. J. Neurochem., 107, 1284-1293 (2008).

18) MacDonald SC, Fleetwood IG, Hochman S, Dodd JG, Cheng GK, Jordan LM, Brownstone RM. Functional motor neurons differentiating from mouse multipotent spinal cord precursor cells in culture and after transplantation into transected sciatic nerve. J. Neurosurg., 98, 1094-1103 (2003).

19) Schnitzler AC, Lopez-Coviella I, Blusztajn JK. Differential modulation of nerve growth factor receptor (p75) and cholinergic gene expression in purified p75-expressing and non-expressing basal forebrain neurons by BMP9. Brain Res., 1246, 19-28 (2008).

20) Akpan N, Caradonna K, Chuenkova MV, PereiraPerrin M. Chagas' disease parasite-derived neurotrophic factor activates cholinergic gene expression in neuronal PC12 cells. Brain Res., 1217, 195-202 (2008).

21) Woolf NJ, Milov AM, Schweitzer ES, Roghani A. Elevation of nerve growth factor and antisense knockdown of TrkA receptor during contextual memory consolidation. J. Neurosci., 21, 10471055 (2001).

22) Terry AV Jr, Buccafusco JJ. The cholinergic hypothesis of age and Alzheimer's disease-related cognitive deficits: recent challenges and their implications for novel drug development. J. Pharmacol. Exp. Ther., 306, 821-827 (2003).

23) Pfeil U, Vollerthun R, Kummer W, Lips KS. Expression of the cholinergic gene locus in the rat placenta. Histochem. Cell Biol., 122, 121-130 (2004).

24) Izant JG, McIntosh J. Microtubule-associated proteins: a monoclonal antibody to MAP-2 binds to differentiated neurons. Proc. Natl. Acad. Sci. U.S.A., 77, 4741-4745 (1980).

25) Wong TP, Debeir T, Duff K, Cuello AC. Reorganization of cholinergic terminals in the cerebral cortex and hippocampus in transgenic mice carrying mutated presenilin-1 and amyloid precursor protein transgenes. J. Neurosci., 19, 2706-2716 (1999).

26) Tian X, Sun X, Suszkiw JB. Developmental age-dependent upregulation of choline acetyltransferase and vesicular acetylcholine transporter mRNA expression in neonatal rat septum by nerve growth factor. Neurosci. Lett., 209, 134-136 (1996).
27) Niewiadomska G, Mietelska-Porowska A, Mazurkiewicz M. The cholinergic system, nerve growth factor and the cytoskeleton. Behav. Brain Res., 221, 515-526 (2011).

28) Wu PY, Lin YC, Chang CL, Lu HT, Chin CH, Hsu TT, Chu D, Sun $\mathrm{SH}$. Functional decreases in $\mathrm{P} 2 \mathrm{X} 7$ receptors are associated with retinoic acid-induced neuronal differentiation of Neuro-2a neuroblastoma cells. Cell. Signal., 21, 881-891 (2009).

29) De Gois S, Houhou L, Oda Y, Corbex M, Pajak F, Thévenot E, Vodjdani G, Mallet J, Berrard S. Is RE1/NRSE a common cisregulatory sequence for ChAT and VAChT genes? J. Biol. Chem., 275, 36683-36690 (2000).

30) Yamaguchi Y, Tabata K, Asami S, Miyake M, Suzuki T. A novel cyclophane compound, CPPy, facilitates NGF-induced TrkA signal transduction and induces cell differentiation in neuroblastoma. Biol. Pharm. Bull., 30, 638-643 (2007).

31) Kippin TE, Martens DJ, van der Kooy D. p21 loss compromises the relative quiescence of forebrain stem cell proliferation leading to exhaustion of their proliferation capacity. Genes Dev., 19, 756-767 (2005).

32) Qiu MS, Green SH. NGF and EGF rapidly activate p21ras in PC12 cells by distinct, convergent pathways involving tyrosine phosphorylation. Neuron, 7, 937-946 (1991).

33) Bruno JG, Carrillo MP, Phillips T, King B. Development of DNA aptamers for cytochemical detection of acetylcholine. In Vitro Cell. Dev. Biol. Anim., 44, 63-72 (2008).

34) Pardridge WM. CNS drug design based on principles of bloodbrain barrier transport. J. Neurochem., 70, 1781-1792 (1998).

35) Odani T, Tanizawa H, Takino Y. Studies on the absorption, distribution, excretion and metabolism of ginseng saponin. II. The absorption, distribution and excretion of ginsenoside Rg1 in the rat. Chem. Pharm. Bull., 31, 292-298 (1983).

36) Tachikawa E, Kudo K. Proof of the mysterious efficacy of ginseng: basic and clinical trials: suppression of adrenal medullary function in vitro by ginseng. J. Pharmacol. Sci., 95, 140-144 (2004).

37) Wu J, Jeong HK, Bulin SE, Kwon SW, Park JH, Bezprozvanny I. Ginsenosides protect striatal neurons in a cellular model of Huntington's disease. J. Neurosci. Res., 87, 1904-1912 (2009).

38) Musende AG, Eberding A, Wood CA, Adomat H, Fazli L, HurtadoColl A, Jia W, Bally MB, Tomlinson Guns ES. A novel oral dosage formulation of the ginsenoside aglycone protopanaxadiol exhibits therapeutic activity against a hormone-insensitive model of prostate cancer. Anticancer Drugs, 23, 543-552 (2012).

39) Hao K, Gong P, Sun SQ, Hao HP, Wang GJ, Dai Y, Liang Y, Xie L, Li FY. Beneficial estrogen-like effects of ginsenoside Rb1, an active component of Panax ginseng, on neural 5-HT disposition and behavioral tasks in ovariectomized mice. Eur. J. Pharmacol., 659, 15-25 (2011).

40) Bora SH, Liu Z, Kecojevic A, Merchenthaler I, Koliatsos VE. Direct, complex effects of estrogens on basal forebrain cholinergic neurons. Exp. Neurol., 194, 506-522 (2005). 